\title{
Hsp27 regulates epithelial mesenchymal transition, metastasis and proliferation in colorectal carcinoma
}

\author{
LU HAN ${ }^{*}$, YONG JIANG ${ }^{*}$, DONGXING HAN and WEILIN TAN \\ Department of Surgery, Shanghai Jiangong Hospital, Shanghai 200083, P.R. China
}

Received September 30, 2015; Accepted January 5, 2018

DOI: $10.3892 / \mathrm{ol} .2018 .9286$

\begin{abstract}
The primary factor associated with poor survival rate in patients with colorectal carcinoma (CRC) is the presence of metastasis. The underlying molecular mechanisms of CRC metastasis are yet to be fully elucidated. The present study investigated the function of heat shock protein 27 (Hsp27) on the invasion and proliferation of CRC cells. The clinical significance of Hsp27 was evaluated using tissue microarray analysis $(n=81)$. Invasion and metastasis assays were used to determine the function of Hsp27 in CRC metastasis in vitro and in vivo using RNA interference and the ectopic expression of Hsp27. The upregulation of Hsp27 has been frequently identified in CRC tissues. Patients with CRC and a high expression level of Hsp27 have a reduced overall survival rate. Silencing Hsp27 inhibited the growth and invasion of CRC cells in vitro and in vivo, whereas ectopic overexpression of Hsp27 promoted the proliferation and invasion of CRC cells in vitro. Furthermore, depletion of Hsp27 expression inhibited the epithelial-to-mesenchymal transition (EMT), whilst ectopic overexpression of Hsp27 induced EMT. The results of the present study indicated that Hsp27 serves an important function in the aggressiveness of CRC through inducing EMT. Hsp27 suppression may represent a potential therapeutic option for the suppression of CRC progression.
\end{abstract}

\section{Introduction}

Colorectal carcinoma $(\mathrm{CRC})$ is the third most common malignant cancer and the second most common cause of cancer-associated mortality globally (1). The quality of life and the 5-year survival rate remain low in patients with CRC, primarily due to the presence of metastasis at the time of

Correspondence to: Dr Weilin Tan, Department of Surgery, Shanghai Jiangong Hospital, 666 Zhongshan North No. 1 Road, Shanghai 200083, P.R. China

E-mail:weilintan88@163.com

*Contributed equally

Key words: heat shock protein 27, epithelial-to-mesenchymal transition, colorectal carcinoma, metastasis initial diagnosis. Although a large number of proteins and signaling pathways have been identified as associated with metastasis in patients with CRC, the underlying molecular mechanisms of CRC metastasis have yet to be fully elucidated (2-4).

Heat shock protein 27 (Hsp27) is a small, ubiquitous heat shock protein, which has an important function in regulating the balance between cell death and survival. Hsp27 expression is induced under stress conditions to allow cells to adapt to cellular stresses, including protein misfolding, aggregation and denaturation (5). Previous studies have established that Hsp27 is an important mediator in cancer progression through the prevention of apoptosis in transformed cells. Cancer tissues have a hypoxic and ischemic microenvironment, so Hsp27 expression is increased in a number of malignancies, including lung (6), prostate (7), gastric (8), breast (9) and CRC (10). Hsp27 inhibition using OGX-427, a targeted inhibitor, may synergistically enhance the activity of numerous chemotherapies (11-13).

Previous studies have established that Hsp27 may also serve an important function in cancer metastasis. Hsp27 promotes migration and invasion in breast cancer cells $(9,14)$. It may mediate the epithelial-to-mesenchymal transition (EMT) in lung cancer cells and kidney fibrosis $(15,16)$. Shiota et al (7) reported that Hsp27 was required for interleukin (IL)-6-mediated EMT via the modulation of signal transducer and activator of transcription 3/Twist signaling. Hsp27 suppression may also be a promising therapeutic strategy for metastasis of prostate cancer $(7,17)$. However, the biological functions of Hsp27 in the development and metastasis of CRC are yet to be fully understood, and the mechanism underlying how Hsp27 exerts its function requires further investigation.

The results of the present study indicated that Hsp27 has an important function in the development of a poor prognostic phenotype in patients with CRC. Overexpression of Hsp27 promoted the invasiveness of CRC cells, whereas the knockdown of Hsp27 in CRC cells suppressed cell invasive and proliferative capacity in in vitro and in vivo experiments. The present study also reported a function for Hsp27 in inducing EMT in CRC cells, which may have implications for metastasis in CRC. Overall, these results suggested that Hsp27 is a predictive factor for the survival outcome of patients with $\mathrm{CRC}$, and that Hsp27 may be a potential therapeutic target for the treatment of CRC metastasis. 


\section{Materials and methods}

Immunohistochemical analysis of tissue microarrays (TMA). TMA were constructed using formalin-fixed, paraffin-embedded tissue samples. Tumor samples were collected from 81 patients with colorectal cancer who underwent surgery at Shanghai Jiangong Hospital (Shanghai, China), from January 2008 to December 2013 and TMAs were constructed by Shanghai Biochip Co., Ltd. (Shanghai, China). Clinicopathological characteristics of CRC patients are presented in Table I. Patients did not receive any treatment prior to surgery. The tumor stage was determined according to the 2010 American Joint Committee on Cancer and International Union Against Cancer tumor-node-metastasis classification system (18). All tumor samples were acquired at the time of operation and fixed with paraformaldehyde (4\%) at room temperature for $24 \mathrm{~h}$. Overall survival was defined as the interval between the date of surgery and mortality. Surviving patients were censored at the last follow up. Ethical approval was obtained from the Research Ethics Committee of Shanghai Jiangong Hospital (Shanghai, China) and written informed consent was obtained from each patient.

Immunostaining was performed on tissue microarray slides with specific primary antibodies against Hsp27. Paraffin sections were deparaffinized in xylene and rehydrated through decreasing concentrations of ethanol (100, 95 and 85\%, 5 min each). Antigens were retrieved by microwave irradiation in a microwave oven for $3 \mathrm{~min}$ in $\mathrm{pH} 6.0$ citric buffer and cooled at room temperature for $60 \mathrm{~min}$. Endogenous peroxidase activity was blocked by incubation of the slides in $3 \% \mathrm{H}_{2} \mathrm{O}_{2} / \mathrm{PBS}$, and nonspecific binding sites were blocked with $10 \%$ goat serum (Thermo Fisher Scientific, Inc). Primary antibodies were diluted as follows: Mouse monoclonal antibody against Hsp27 (cat. no. 2402; 1:200; Cell Signaling Technology, Inc., Danvers, MA, USA). An EnVision Detection kit (GK500705: Gene Tech, Shanghai, China) was used to visualize tissue antigens. Immunohistochemical score was independently assessed by 2 blinded pathologists (Shanghai Jiangong Hospital, Shanghai, China). The percentage of positive cells was divided into 4 grades: $0-25,1 ; 26-50,2 ; 51-75,3$; and $>75 \%$, 4 . The intensity of the staining was divided into 4 grades (intensity scores): No staining, 0; light brown, 1; brown, 2; and dark brown, 3. The immunostaining score was evaluated on the basis of percentage score $\mathrm{x}$ intensity score. Overall scores of 0-6 or 7-12 were defined as weak or strong staining, respectively. The integrated optical density was measured using Image-Pro Plus v6.0 software (Media Cybernetics, Inc., Rockville, MD, USA).

Cell culture. The HT29, HCT116 and LOVO CRC cell lines were obtained from the American Type Culture Collection (Manassas, VA, USA). The cells were cultured in Dulbecco's modified Eagle's medium (DMEM; Thermo Fisher Scientific, Inc) supplemented with $10 \%$ fetal bovine serum (FBS; Thermo Fisher Scientific, Inc) and antibiotics (100 U/ml penicillin, $100 \mathrm{mg} / \mathrm{ml} \mathrm{streptomycin),} \mathrm{and} \mathrm{cultured} \mathrm{in} \mathrm{an} \mathrm{atmosphere}$ containing $5 \% \mathrm{CO}_{2}$ at $37^{\circ} \mathrm{C}$.

Gene constructs, lentivirus production and transfection. The Hsp27-RNA interference lentiviral vector was constructed by GeneChem Co., Ltd., (Shanghai, China). A total of
3 double-stranded oligonucleotides specifically targeted to Hsp27 mRNA were inserted into the short hairpin (sh)RNA expression plasmid vector pGCSIL-green fluorescent protein (GFP). The cDNA encoding Hsp27 was amplified using polymerase chain reaction (PCR) and cloned into the pGC-FU-GFP vector. The lentivirus was generated and harvested as described previously (19). Cells were seeded in 6-well platesat a density of $2 \times 10^{4} /$ well, and $2 \mathrm{ml} \mathrm{DMEM}$ with $1 \%$ penicillin-streptomycin and $10 \%$ FBS was added to each well. Viral particles at a multiplicity of infection (MOI) of 2.5,5, 10, 20,50, and 100 were added to the wells. Following incubation at $37^{\circ} \mathrm{C}$ in $5 \% \mathrm{CO}_{2}$ for $24 \mathrm{~h}$, the virus-containing medium was removed and replaced with $2 \mathrm{ml}$ fresh culture medium per well. The transduction efficiency was determined daily with an inverted fluorescent microscope and was quantified by measuring the GFP-expressing cells as a percentage of the total number of visible cells. Control cells were transfected with an empty control vector.

Reverse transcription-quantitative $(R T-q) P C R$. Total RNA was extracted from cells and tissues using TRIzol reagent (Thermo Fisher Scientific, Inc., Waltham, MA, USA). Total RNA was reverse transcribed using RevertAid First-Strand cDNA Synthesis kit (Thermo Fisher Scientific, Inc.). Hsp27 mRNA levels were determined by qPCR using SYBR Premix Ex Taq (Takara Biotechnology Co., Ltd., Dalian, China). Then Set up the experiment and the following PCR program: $1.50^{\circ} \mathrm{C}$ $2 \mathrm{~min}, 1$ cycle; $2.95^{\circ} \mathrm{C} 10 \mathrm{~min}, 1$ cycle; $3.95^{\circ} \mathrm{C} 15 \mathrm{sec}, 60^{\circ} \mathrm{C}$ $30 \mathrm{sec}, 72^{\circ} \mathrm{C} 30 \mathrm{sec}$, for 40 cycles; $4.72^{\circ} \mathrm{C} 10 \mathrm{~min}, 1$ cycle. The expression levels were normalized against GAPDH. The relative expression level of Hsp27 was determined using the following equation: $2^{-\Delta \Delta \mathrm{Cq}}\left(\Delta \mathrm{Cq}=\Delta \mathrm{Cq}^{\text {target }}-\Delta \mathrm{Cq}^{\mathrm{GAPDH}}\right)(20)$. RT-qPCR primer sequences are listed as following: Hsp27, forward, ACGGTCAAGACCAAGGATGG and reverse, AGGGAGGAGGAAACTTGGT; E-cadherin, forward, CAC CTGGAGAGAGGCCATGT and reverse, TGGGAAACA TGAGCAGCTCT; n-cadherin, forward, ATGTGCCGGATA GCGGGAGC and reverse, TACACCGTGCCGTCCTCGTC; vimentin, forward, CTTGAACGGAAAGTGGAATCCT and reverse, GTCAGGCTTGGAAACGTCC; ZO-1, forward, AAGTCACACTGGTGAAATCC and reverse, CTCTTGCTG CCAAACTATCT; GAPDH, forward, CGTATTGGGCGC CTGGTCAC and reverse, ATGATGACCCTTTTGGCTCC.

Western blot analysis. Western blot analysis was performed as previously reported (21). Briefly, cell lysates were prepared with a Tissue-Protein Extraction reagent (Thermo Fisher Scientific, Inc.). The concentration of total protein was determined using a bicinchoninic acid assay kit, according to the manufacturer's protocol (Thermo Fisher Scientific, Inc.). Proteins were separated by SDS-PAGE and transferred onto polyvinylidene difluoride membranes. The membranes were washed, blocked, and incubated with anti-Hsp27 (cat. no. 2402; dilution, 1:1,000; Cell Signaling Technology, Inc., Danvers, MA, USA), anti-E-cadherin (cat. no. 3195; dilution, 1:1,000; Cell Signaling Technology, Inc.), anti-N-cadherin (cat. no. 4061; dilution, 1:1,000; Cell Signaling Technology, Inc.), anti-Vimentin (cat. no. ab24525; dilution, 1:200; Abcam, Cambridge, UK), anti-zonula occludens 1 (cat. no. ab96587; ZO-1; dilution, 1:200; Abcam) or anti-GAPDH (cat. no. G8795; dilution, 1:5,000; Sigma-Aldrich; Merck KGaA, Darmstadt, Germany) overnight at $4^{\circ} \mathrm{C}$, then probed with 

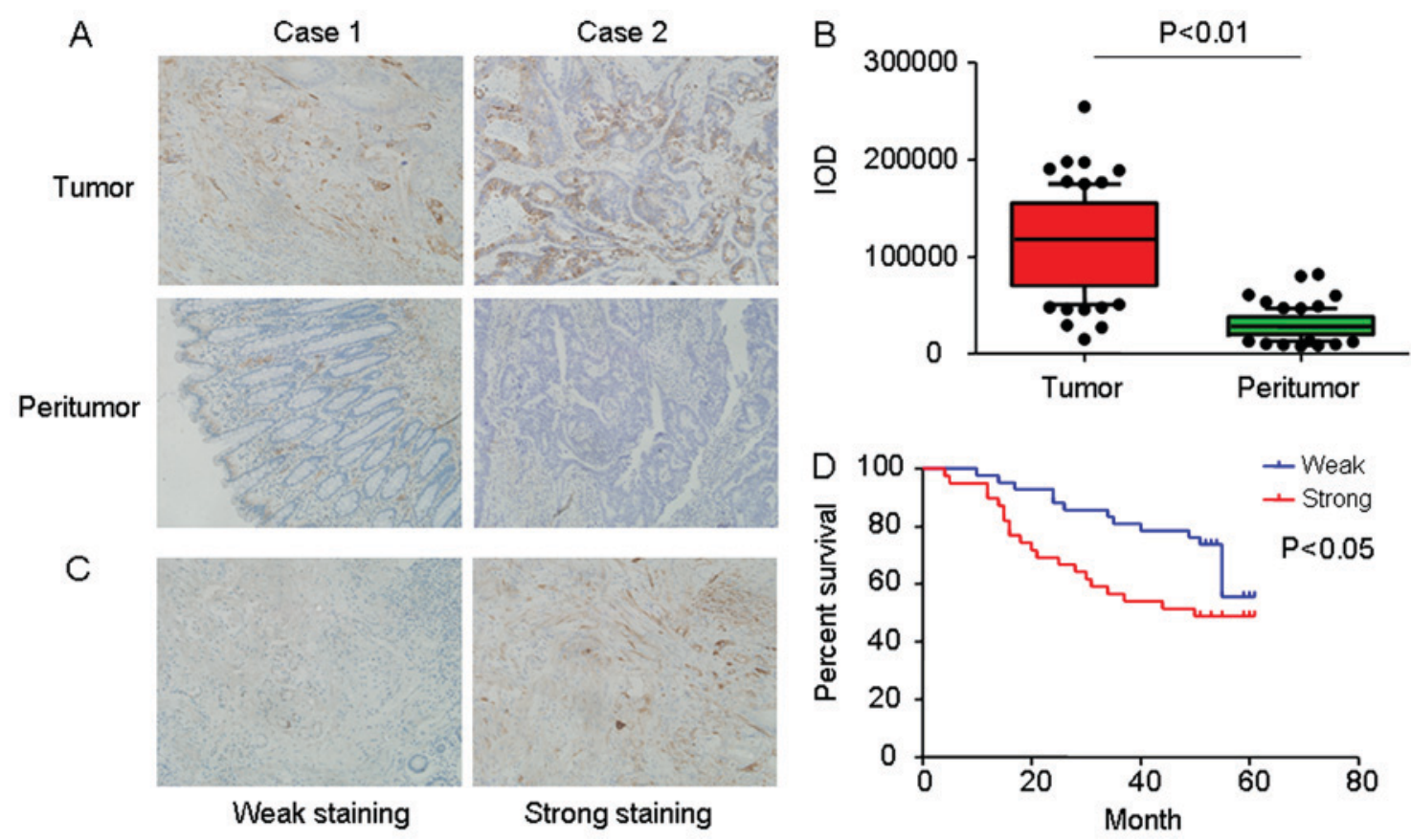

Figure 1. Expression of Hsp27 in CRC tissues. (A) Representative immunohistochemical images of Hsp27 staining in matched tumor tissues and adjacent normal colorectal mucosal tissue (magnification, x400). (B) A box and whisker plot (whiskers: 10-90\%) of IOD for Hsp27 was obtained from TMA. (C) Representative expression levels of Hsp27 in CRC TMA, analyzed using immunochemistry analysis (weak staining, score + and negative; strong staining, score ++ and +++. Magnification, x400). (D) Kaplan-Meier analysis of overall survival in 81 patients with CRC based on Hsp27 expression. Hsp27, heat shock protein 27; CRC, colorectal carcinoma; IOD, integrated optical density; TMA, tumor microarray.

secondary antibodies [horseradish peroxidase (HRP)-conjugated goat anti-mouse IgG; cat. no. 1706516 or HRP-conjugated goat anti-rabbit IgG; cat. no. 1706515; 1:20,000; Bio-Rad Laboratories, Inc.] at room temperature for $1 \mathrm{~h}$. The bands of the proteins were detected using enhanced chemiluminescence detection (Bio-Rad Laboratories, Inc.).

Cell Counting Kit-8 (CCK-8) assay. Cell proliferation was determined using a CCK- 8 assay. Cells were seeded at a density of $2 \times 10^{3}$ cells/well in 96-well plates, which were cultured in $100 \mu \mathrm{l}$ DMEM. Following culture for 24, 48, 72 or 96 h, $10 \mu$ l CCK-8 (Dojindo Molecular Technologies, Inc., Kumamoto, Japan) was added to each well, and then the 96-well plates were incubated at $37^{\circ} \mathrm{C}$ for $4 \mathrm{~h}$. The staining intensity of the medium was measured at a wavelength of $450 \mathrm{~nm}$ using a microplate reader.

Cell invasion assays. The cell invasion assays were conducted using an $8-\mu \mathrm{m}$ pore size Transwell chamber (Corning Incorporated, Corning, NY, USA) coated with Matrigel. DMEM supplemented with $10 \%$ FBS was added to the lower chamber as a chemoattractant. Cells $\left(5 \times 10^{4}\right)$ were suspended in DMEM supplemented with $1 \%$ FBS and plated into the upper chamber. Following incubation for $48 \mathrm{~h}$, the cells on the bottom were fixed with paraformaldehyde (4\%) at room temperature for $1 \mathrm{~h}$ and stained using crystal violet $(0.05 \% \mathrm{w} / \mathrm{v})$ for $1 \mathrm{~h}$. The invasive cells were imaged at $\times 200$ magnification and counted using a light microscope (5 fields/group).

Xenograft mouse model. Animal studies were approved by the Animal Ethics Committee of Shanghai Jiangong hospital. All of the mice $(n=12)$ were purchased from Shanghai SLAC Laboratory Animal Co., Ltd., and housed according to the protocols approved by the Shanghai Medical Experimental Animal Care Commission (Shanghai, China). HCT116 cells (with stably silenced Hsp27 expression or mock cells; $1 \times 10^{7}$ cells) were suspended in $100 \mu \mathrm{l}$ serum-free DMEM and subcutaneously injected into the upper flank of each mouse (6 per group, male BALB/c-nu/nu, 8 weeks old, the average weight of these mice were $20 \mathrm{~g}$ ). Tumor growth was measured each week. Then, the mice were sacrificed on day 30 following the cell injection. At necropsy, the tumors were analyzed for largest (a) and smallest (b) diameters, and the tumor volume was calculated as $\mathrm{V}=\mathrm{a} \mathrm{xb}^{2} / 2$.

For the lateral tail vein metastasis model, $2 \times 10^{6}$ HCT116-Mock and HCT116-sh-Hsp27 cells were suspended in $200 \mu \mathrm{l}$ serum-free DMEM and injected into the lateral tail vein of the nude mice. All the mice were sacrificed on day 40 following the cell injection. In vivo tumor formation and metastases were analyzed using hematoxylin and eosin staining.

Statistical analysis. Data were represented as the mean \pm standard error of the mean of $\geq 3$ independent experiments. Statistical analysis was performed using SPSS v15.0 for Windows (SPSS, Inc., Chicago, IL, USA). Kaplan-Meier analysis was performed for survival analysis. Quantitative variables were analyzed using the Student's t-test. $\mathrm{P}<0.05$ was considered to indicate a statistically significant difference.

\section{Results}

Hsp27 is frequently overexpressed in human CRC and predicts poor prognosis for patients with CRC. To investigate the function of Hsp27 in CRC, the expression of Hsp27 in human CRCs 

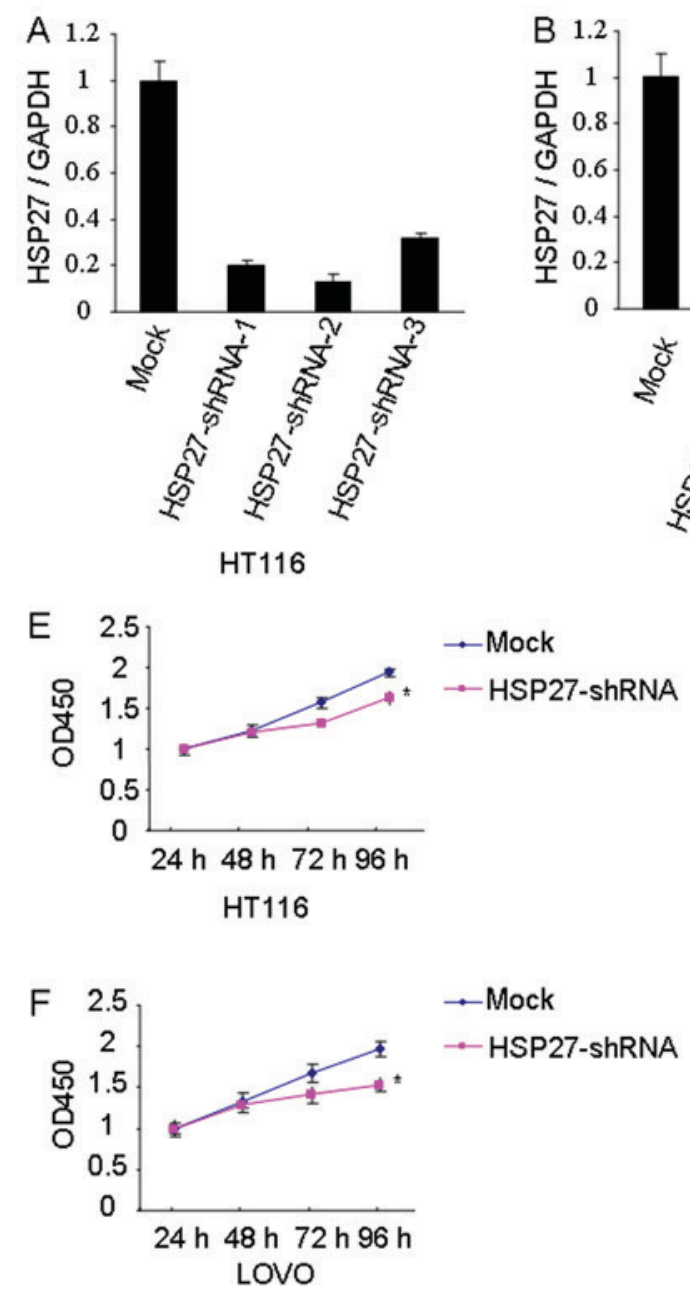
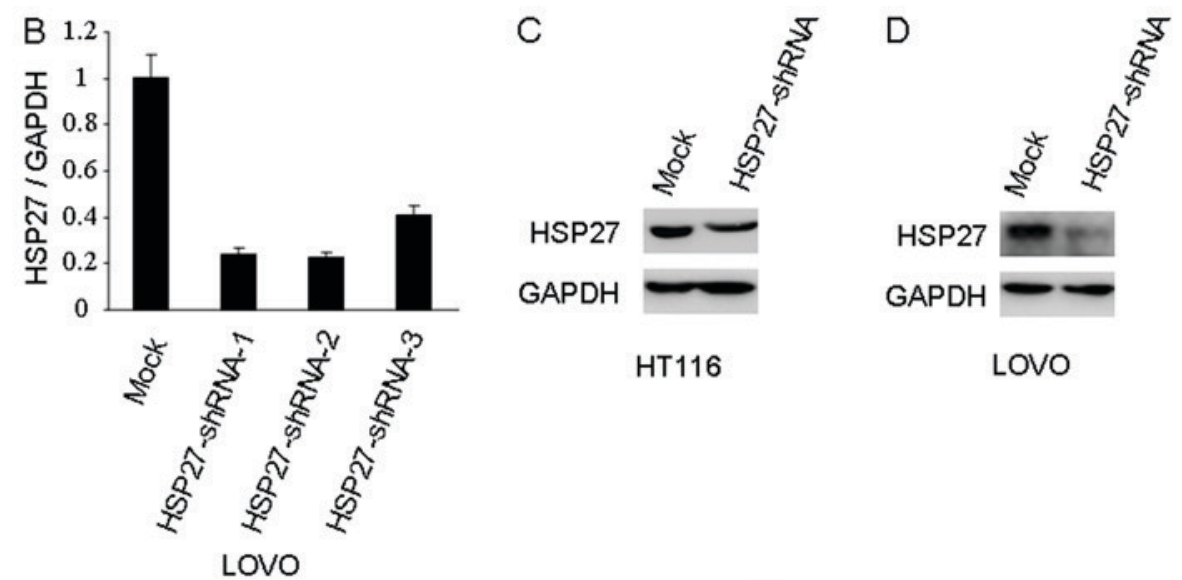

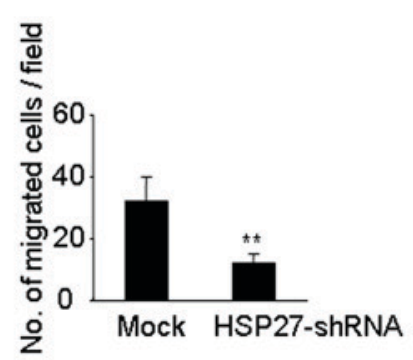

HT116
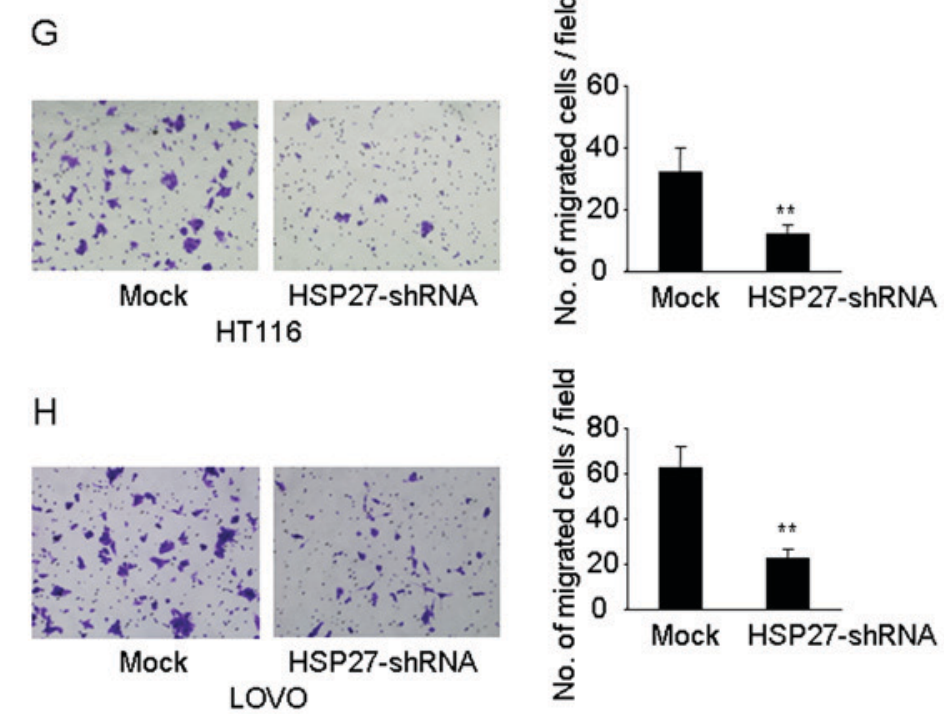

Figure 2. Downregulation of Hsp27 inhibits proliferation and invasion of colorectal carcinoma cells. Efficient knockdown of Hsp27 was determined using reverse transcription-quantitative polymerase chain reaction in (A) HT116 and (B) LOVO cells, and western blotting in (C) HT116 and (D) LOVO cells. Hsp27 silencing significantly inhibited cell proliferation in (E) HCT116 and (F) LOVO cells. shRNA-mediated suppression of Hsp27 mediated significant reduction of $(\mathrm{G})$ HCT116 and (H) LOVO cell invasion (magnification, $\mathrm{x} 200$ ). ${ }^{*} \mathrm{P}<0.05,{ }^{* *} \mathrm{P}<0.01$ vs. mock. Hsp27, heat shock protein 27 ; shRNA, short hairpin RNA.

was evaluated. Immunohistochemical staining for Hsp27 was performed using tissue microarrays, which contained 81 pairs of CRC specimens and corresponding normal colorectal mucosal tissues. Representative images of Hsp27 expression are presented in Fig. 1A. The immunohistochemistry results indicated that $\mathrm{Hsp} 27$ was frequently increased in CRC specimens compared with corresponding normal colorectal mucosal tissues $(\mathrm{P}<0.01$; Fig. 1B).

In addition, the prognostic value of $\mathrm{Hsp} 27$ in patients with $\mathrm{CRC}$ was investigated. Levels of $\mathrm{Hsp} 27$ protein in tumor tissues were classified as strong expression in 39 patients $(39 / 81$, $48.1 \%$ ) and weak expression in 42 patients (42/81, 51.9\%). Representative images of Hsp27 expression were presented in Fig. 1C. Kaplan-Meier analysis identified that the mean overall survival time for patients with overexpression of Hsp27 was 50.00 months compared with patients with a low expression of Hsp27 (log-rank test, $\mathrm{P}=0.031$; Fig. 1D).

Hsp27 enhances cell migration and invasion in CRC in vitro. The results of the present study have demonstrated that Hsp27 is frequently overexpressed in patients with CRC and associated with poor prognosis. Therefore, it is possible that Hsp27 may be involved in CRC metastasis. Lentivirus-mediated shRNA was used to knock down the expression of Hsp27 in HCT116 and LOVO cells. The knockdown efficiency was verified using western blot and RT-qPCR (Fig. 2A-D). As presented in Fig. 2E-F, the knockdown of Hsp27 significantly inhibited cell proliferation of HT116 and LOVO cells, as measured by CCK-8 assay $(\mathrm{P}<0.05$; Fig. $2 \mathrm{E}$ and $\mathrm{F}$, respectively). Matrigel invasion assays identified that decreased expression of Hsp27 impaired the invasive abilities of HCT116 and LOVO cell lines ( $\mathrm{P}<0.01$; Fig. $2 \mathrm{G}$ and $\mathrm{H}$, respectively).

To further investigate the effect of Hsp27 on CRC progression, Hsp27 was stably expressed in HT29 cells, which have a low expression level of endogenous Hsp27. The effective overexpression of Hsp27 in HT29 cells was confirmed using RT-qPCR and western blotting (Fig. 3A and B, respectively). Overexpression of Hsp27 promoted cell proliferation of HT29 cells (Fig. 3C). The Matrigel invasion assay demonstrated that overexpression of Hsp27 markedly increased the invasive potential of HT29 cells compared with mock cells $(\mathrm{P}<0.05$; Fig. 3D and E). Collectively, these results indicated the functional significance of Hsp27 in promoting an aggressive phenotype in CRC cells. 


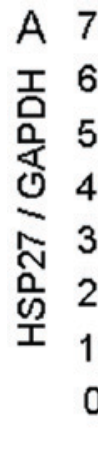

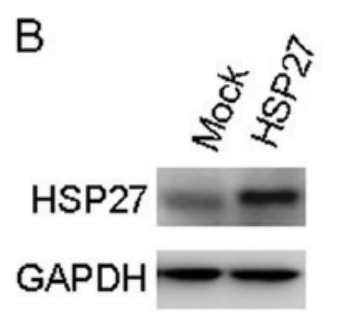
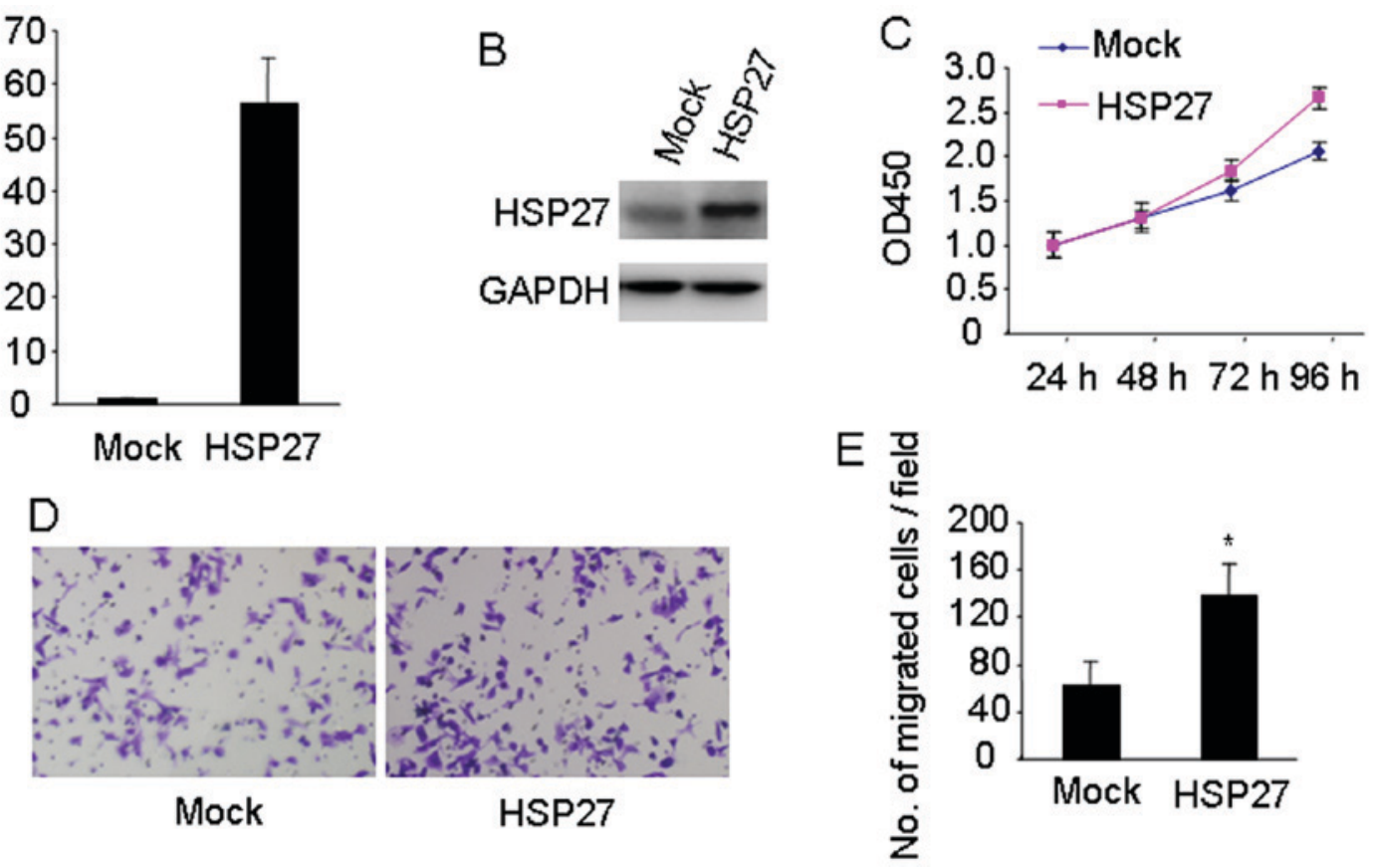

Figure 3. Upregulation of Hsp27 promotes the proliferation and invasion of colorectal carcinoma cells. Effective overexpression of Hsp27 in HT29 cells was confirmed using (A) reverse transcription-quantitative polymerase chain reaction and (B) western blot analysis. (C) Overexpression of Hsp27 in HT29 cells increased cell proliferation, measured using a Cell Counting Kit-8 assay. (D) Matrigel invasion assay demonstrated that Hsp27-overexpressed in HT29 cells exhibited increased invasive ability compared with mock cells with (E) quantification (magnification, $\mathrm{x} 200$ ). ${ }^{*} \mathrm{P}<0.05$. Hsp27, heat shock protein 27.

Expression levels of Hsp27 influence EMT in CRC cell lines. The EMT is the series of events that converts epithelial cells into migratory cells, and it is associated with tumor metastasis. Therefore, the involvement of EMT in the Hsp27-mediated alteration of invasive potential of CRC cells was investigated. The alteration of EMT markers between Hsp27-shRNA and their parental cells was investigated using RT-qPCR and western blotting. The silencing of Hsp27 in CRC cells induced the expression of E-Cad and ZO-1 epithelial markers, and this was accompanied by a concomitant reduction of the mesenchymal markers, N-Cad and Vimentin (Fig. 4). Therefore, these data suggested a loss of mesenchymal-like features and reacquisition of epithelial characteristics in Hsp27-depleted CRC cells.

However, HT29 cells exhibited a downregulation of E-cadherin and ZO-1, and an upregulation of N-Cad and Vimentin following the ectopic overexpression of Hsp27, as assessed by RT-qPCR and western blotting analysis (Fig. 4). These results indicated that the expression levels of Hsp27 may influence the EMT of CRC cells in vitro.

Hsp27 promotes CRC progression and metastasis in vivo. To investigate the involvement of Hsp27 in CRC proliferation in vivo, HCT116 cells were treated with a lentivirus targeting Hsp27 to create a HCT116-shRNA cell line that stably downregulated Hsp27 compared with the parental cell line. The HCT116 cells $\left(1 \times 10^{7}\right)$ with stably silenced Hsp27 expression or mock cells were suspended in $100 \mu \mathrm{l}$ serum-free DMEM and then subcutaneously injected into the upper flank of 6 mice for each condition. The comparison of the tumors from the Hsp27 shRNA group and mock group are presented in Fig. 5A-C. The tumor volume in the control group was significantly increased compared with that in the Hsp27 shRNA group $(\mathrm{P}<0.01)$.
For the lateral tail vein metastasis model, the number of lung metastatic nodules was significantly higher in mice injected with HCT116 cells compared with those in mice injected with Hsp27-shRNA HCT116 cells $(\mathrm{P}<0.01)$. These in vivo experiments suggested a function for Hsp27 in the promotion of CRC metastasis (Fig. 5D and E).

\section{Discussion}

Hsp27 expression is induced by heat shock and environmental or pathophysiological stresses, and Hsp27 is a multidimensional protein that functions as a protein chaperone and an antioxidant (22). This protein serves an important function in cell survival and actin cytoskeletal remodeling (22). In numerous types of cancer, Hsp27 is upregulated and associated with poor prognosis $(6,23)$. It is also involved in treatment resistance by protecting cells from therapeutic stressors, including androgen or estrogen withdrawal (13), radiation (24) and cytotoxic chemotherapy $(11,12)$. However, the effect of Hsp27 expression on the survival of patients with CRC and the underlying molecular mechanism by which HSP27 regulates CRC cell aggressiveness remains to be elucidated.

Previous studies have typically focused on the function of Hsp27 in the inhibition of apoptosis. It has been reported that Hsp27 also has an important function in cancer metastasis $(14,17)$. The association between Hsp27 and cancer metastasis has been widely investigated in numerous types of cancer, including HCC $(25,26)$, prostate cancer (7), esophageal squamous cell carcinoma (27) and breast cancer (9). However, the biological functions of Hsp27 in $\mathrm{CRC}$ metastasis require further investigation. In the present study, the protein levels of Hsp27 were analyzed using a CRC TMA containing 81 pairs of CRC tissues (carcinomatous 

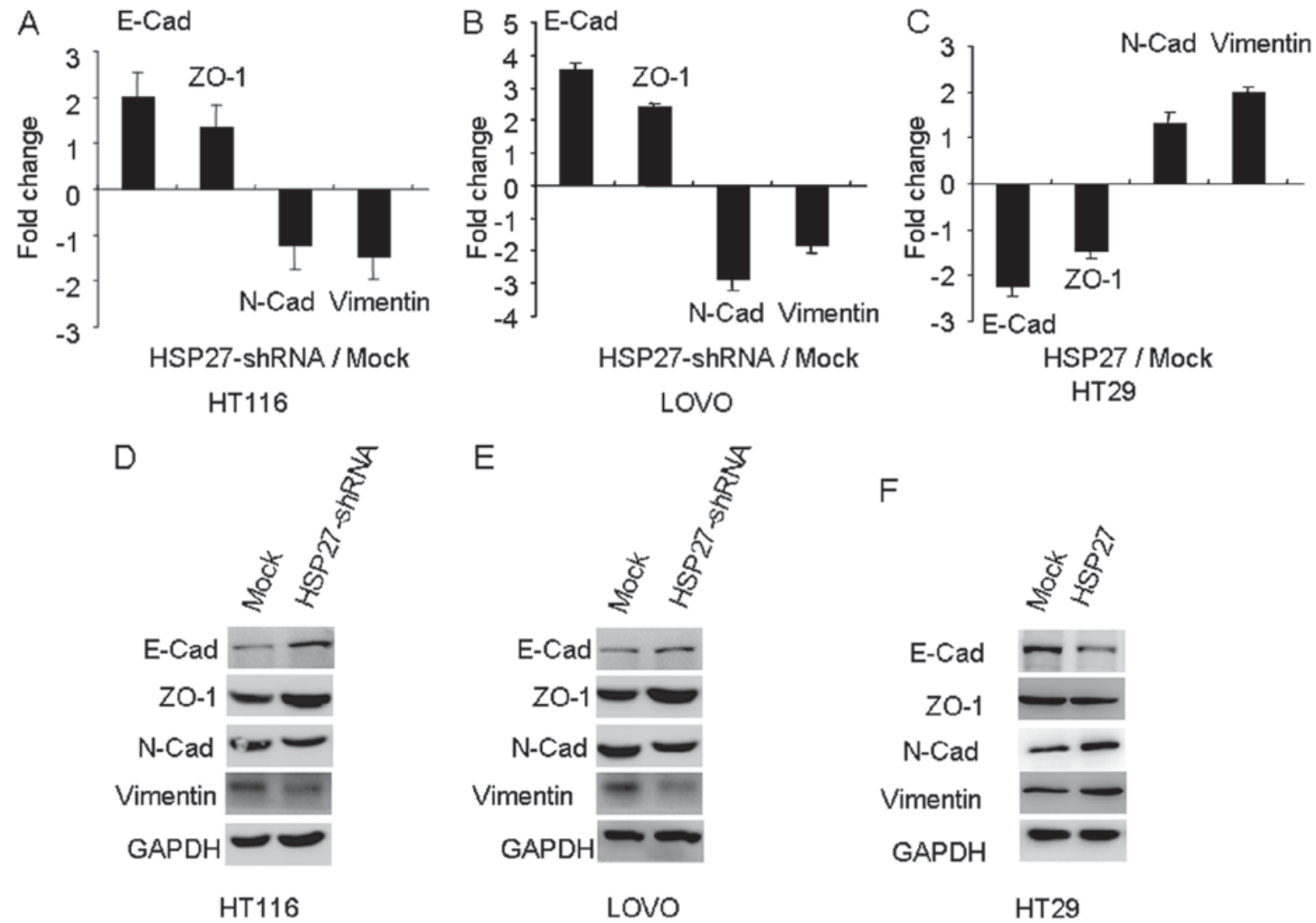

Figure 4. Hsp27 promotes metastasis by inducing epithelial-to-mesenchymal transition. Expression levels of epithelial markers (E-Cad and ZO-1) and mesenchymal markers (N-Cad and Vimentin) were determined using quantitative-polymerase chain reaction analysis in Hsp27-silenced (A) HCT116 and (B) LOVO cells, as well as in (C) Hsp27-overexpressing HT29 cells. The expression of epithelial markers (E-Cad and ZO-1) and mesenchymal markers (N-Cad and Vimentin) were also compared in Hsp27-silenced (D) HCT116 and (E) LOVO cells, as well as (F) Hsp27-overexpressing HT29 cells, using western blot analysis. Hsp27, heat shock protein 27; ZO-1, zonula occludens 1; E-Cad, E-cadherin; N-Cad, N-cadherin; shRNA, short hairpin RNA.

A

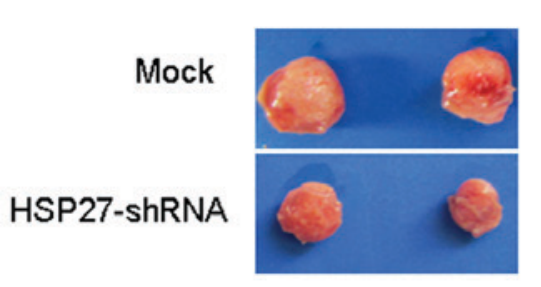

$\overline{1 \mathrm{~cm}}$
B

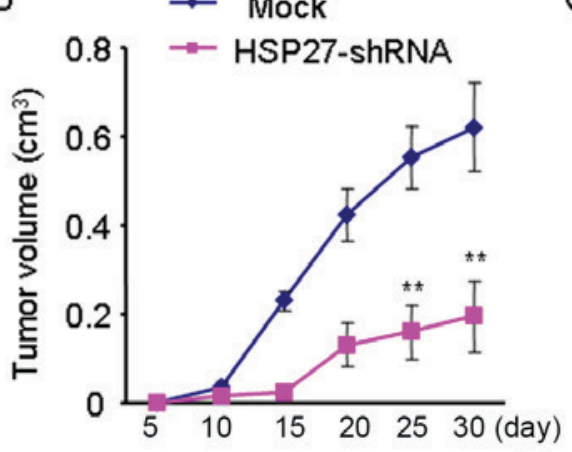

$\mathrm{C}$

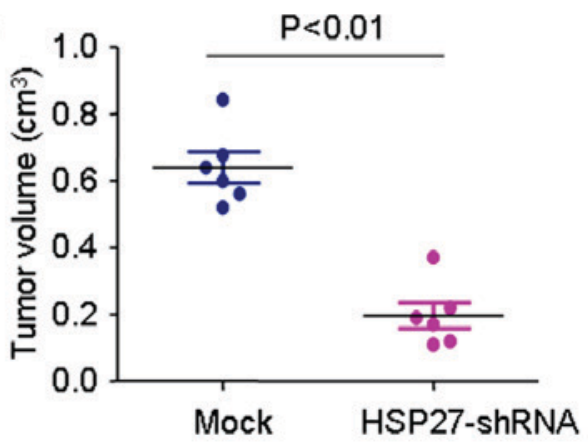

$\mathrm{D}$

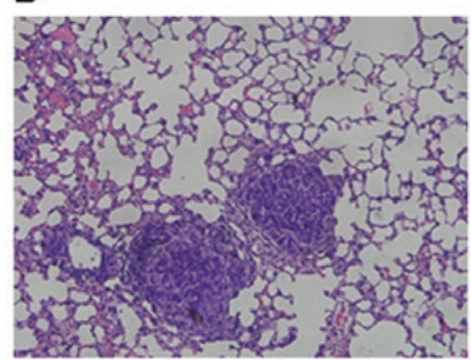

Mock

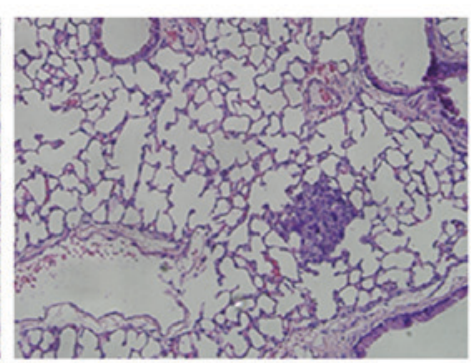

HSP27-shRNA
$\mathrm{E}$

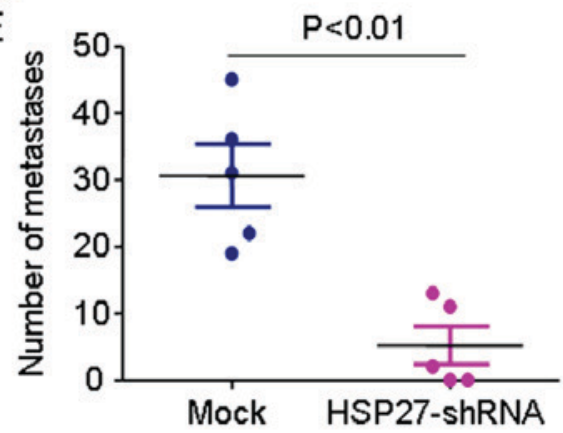

Figure 5. Hsp27 promotes colorectal cancer progression and metastasis in vivo. (A) Representative tumors from the mock and Hsp27-shRNA groups. Depletion of Hsp27 expression in HCT116 cells significantly inhibited tumor growth in vivo, evidenced by (B) growth curve of tumor and (C) tumor volume. Knockdown of Hsp27 expression inhibited lung metastasis in an experimental mouse model, evidenced by (D) representative images (magnification, $\mathrm{x} 400)$ and (E) quantification. ${ }^{* *} \mathrm{P}<0.01$ vs. mock. Hsp27, heat shock protein 27 ; shRNA, short hairpin RNA. 
and non-neoplastic human colorectal tissues). These results indicated that the staining density of Hsp27 was increased compared with that observed in non-neoplastic human colorectal tissues. In addition, the Kaplan-Meier analysis demonstrated that high levels of Hsp27 are associated with poor prognosis in patients with CRC. These data indicated that Hsp27 may be an important mediator in the metastasis and invasion of CRC. Therefore, the overexpression of Hsp27 may promote the proliferation and invasion of CRC cells in vitro. However, shRNA-mediated gene suppression of Hsp27 induced a marked reduction in cell proliferation and invasion. It was therefore hypothesized that Hsp27 may be a prognostic factor for patients with CRC and have a pro-metastatic function in CRC. These results identify a potential function of $\mathrm{Hsp} 27$ as part of an underlying biological mechanism in the progression of CRC.

Cordonnier et al (17) demonstrated that Hsp27 regulated $\mathrm{EGF} / \beta$-catenin-mediated EMT in prostate cancer. Shiota et al (7) reported that $\mathrm{Hsp} 27$ is a regulator of IL-6-dependent and -independent EMT, and this was concordant with this chaperone being a therapeutic target to treat metastatic prostate cancer. Pavan et al (28) indicated that Hsp27 is required for the invasion and metastasis promoted by hepatocyte growth factor. Furthermore, Hsp27 participates in the maintenance of breast cancer stem cells via the regulation of EMT and nuclear factor- $\kappa \mathrm{B}$ (14). However, the exact underlying mechanism of how Hsp27 functions in CRC metastasis remains to be determined. An important result in the present study is that Hsp27 was associated with EMT in CRC, and may independently drive EMT. This may be associated with its function in cell invasion and metastasis. The results of the present study demonstrated that Hsp27 silencing increased the expression of E-Cad and ZO-1 epithelial markers and reduces the expression of the mesenchymal markers, N-Cad and Vimentin. In Hsp27-overexpressing HT29 cells, increased expression levels of mesenchymal markers (N-Cad and Vimentin) were observed, as well as an increased invasive ability. As EMT has an important function in cancer invasion and metastasis, these data are concordant with a function for Hsp27 in CRC metastasis.

In conclusion, the present study identified the involvement of Hsp27 in EMT and suggested that Hsp27 has an oncogenic function in CRC progression. Furthermore, these results demonstrated that the overexpression of Hsp27 in CRC is a potential indicator for aggressiveness in tumors and a poor clinical outcome. Therefore, Hsp27 may represent a novel therapeutic target for the management of CRC metastasis.

\section{Acknowledgements}

Not applicable.

\section{Funding}

The present study was supported by a grant from the Jiangong Hospital, Shanghai, China (grant no. 2014-03).

\section{Availability of data and materials}

All data generated or analyzed during this study are included in this published article.

\section{Authors' contributions}

LH and YJ were responsible for data acquisition and analysis and drafting the article; DH performed the in vivo experiments; and WT were responsible for the conception, design, and supervision of the study. All contributing authors gave final approval for the version to be published.

\section{Ethics approval and consent to participate}

Ethical approval was obtained from the Research Ethics Committee of Shanghai Jiangong Hospital (Shanghai, China). Written informed consent was obtained from each patient.

\section{Patient consent for publication}

Written informed consent was obtained from each patient for the publication of this data.

\section{Competing interests}

The authors declare that they have no competing interests.

\section{References}

1. Zhu W, Cai MY, Tong ZT, Dong SS, Mai SJ, Liao YJ, Bian XW, Lin MC, Kung HF, Zeng YX, et al: Overexpression of EIF5A2 promotes colorectal carcinoma cell aggressiveness by upregulating MTA1 through C-myc to induce epithelial-mesenchymaltransition. Gut 61: 562-575, 2012.

2. Urosevic J, Garcia-Albeniz X, Planet E, Real S, Céspedes MV, Guiu M, Fernandez E, Bellmunt A, Gawrzak S, Pavlovic M, et al: Colon cancer cells colonize the lung from established liver metastases through p38 MAPK signalling and PTHLH. Nat Cell Biol 16: 685-694, 2014.

3. Chen DL, Wang ZQ, Zeng ZL, Wu WJ, Zhang DS, Luo HY, Wang F, Qiu MZ, Wang DS, Ren C, et al: Identification of microRNA-214 as a negative regulator of colorectal cancer liver metastasis by way of regulation of fibroblast growth factor receptor 1 expression. Hepatology 60: 598-609, 2014.

4. Yu J, Wu WK, Li X, He J, Li XX, Ng SS, Yu C, Gao Z, Yang J, $\mathrm{Li}$ M, et al: Novel recurrently mutated genes and a prognostic mutation signature in colorectal cancer. Gut 64: 636-645, 2015.

5. Acunzo J, Katsogiannou M and Rocchi P: Small heat shock proteins HSP27 (HspB1), $\alpha$ B-crystallin (HspB5) and HSP22 (HspB8) as regulators of cell death. Int J Biochem Cell Biol 44: 1622-1631, 2012.

6. Zhao GY, Ding JY, Lu CL, Lin ZW and Guo J: The overexpression of 14-3-3 5 and Hsp27 promotes non-small cell lung cancer progression. Cancer 120: 652-663, 2014.

7. Shiota M, Bishop JL, Nip KM, Zardan A, Takeuchi A, Cordonnier T, Beraldi E, Bazov J, Fazli L, Chi K, et al: Hsp27 regulates epithelial mesenchymal transition, metastasis, and circulating tumor cells in prostate cancer. Cancer Res 73: 3109-3119, 2013.

8. Huang Q, Ye J, Huang Q, Chen W, Wang L, Lin W, Lin J and Lin X: Heat shock protein 27 is over-expressed in tumor tissues and increased in sera of patients with gastric adenocarcinoma. Clin Chem Lab Med 48: 263-269, 2010.

9. Gibert B, Eckel B, Gonin V, Goldschneider D, Fombonne J, Deux B, Mehlen P, Arrigo AP, Clézardin P and Diaz-Latoud C: Targeting heat shock protein 27 (HspB1) interferes with bone metastasis and tumour formation in vivo. Br J Cancer 107: 63-70, 2012.

10. Liu W, Ma Y, Huang L, Peng J, Zhang P, Zhang H, Chen J and Qin H: Identification of HSP27 as a potential tumor marker for colorectal cancer by the two-dimensional polyacrylamide gel electrophoresis. Mol Biol Rep 37: 3207-3216, 2010.

11. Baylot V, Andrieu C, Katsogiannou M, Taieb D, Garcia S, Giusiano S, Acunzo J, Iovanna J, Gleave M, Garrido C and Rocchi P: OGX-427 inhibits tumor progression and enhances gemcitabine chemotherapy in pancreatic cancer. Cell Death Dis 2: e221, 2011. 
12. Lamoureux F, Thomas C, Yin MJ, Fazli L, Zoubeidi A and Gleave ME: Suppression of heat shock protein 27 using OGX-427 induces endoplasmic reticulum stress and potentiates heat shock protein 90 inhibitors to delay castrate-resistant prostate cancer. Eur Urol 66: 145-155, 2014.

13. Andrieu C, Taieb D, Baylot V, Ettinger S, Soubeyran P, De-Thonel A, Nelson C, Garrido C, So A, Fazli L, et al: Heat shock protein 27 confers resistance to androgen ablation and chemotherapy in prostate cancer cells through eIF4E. Oncogene 29: 1883-1896, 2010.

14. Wei L, Liu TT, Wang HH, Hong HM, Yu AL, Feng HP and Chang WW: Hsp27 participates in the maintenance of breast cancer stem cells through regulation of epithelial-mesenchymal transition and nuclear factor- $\kappa \mathrm{B}$. Breast Cancer Res 13: R101, 2011.

15. Wettstein G, Bellaye PS, Kolb M, Hammann A, Crestani B, Soler P, Marchal-Somme J, Hazoume A, Gauldie J, Gunther A, et al: Inhibition of HSP27 blocks fibrosis development and EMT features by promoting Snail degradation. FASEB J 27 $1549-1560,2013$.

16. Mizutani H, Okano T, Minegishi Y, Matsuda K, Sudoh J, Kitamura K, Noro R, Soeno C, Yoshimura A, Seike M and Gemma A: HSP27 modulates epithelial to mesenchymal transition of lung cancer cells in a Smad-independent manner. Oncol Lett 1: 1011-1016, 2010.

17. Cordonnier T, Bishop JL, Shiota M, Nip KM, Thaper D, Vahid S, Heroux D, Gleave M and Zoubeidi A: Hsp27 regulates $\mathrm{EGF} / \beta$-catenin mediated epithelial to mesenchymal transition in prostate cancer. Int J Cancer 136: E496-E507, 2015.

18. Edge SB and Compton CC: The American Joint Committee on Cancer: The 7th edition of the AJCC cancer staging manual and the future of TNM. Ann Surg Oncol 17: 1471-1474, 2010.

19. Nasri M, Karimi A and Allahbakhshian Farsani M: Production, purification and titration of a lentivirus-based vector for gene delivery purposes. Cytotechnology 66: 1031-1038, 2014.

20. Livak KJ and Schmittgen TD: Analysis of relative gene expression data using real-time quantitative PCR and the 2(-Delta Delta C(T)) method. Methods 25: 402-408, 2001
21. Li Y, Xu D, Bao C, Zhang Y, Chen D, Zhao F, Ding J, Liang L, Wang Q, Liu L, et al: MicroRNA-135b, a HSF1 target, promotes tumor invasion and metastasis by regulating RECK and EVI5 in hepatocellular carcinoma. Oncotarget 6: 2421-2433, 2015.

22. Acunzo J, Andrieu C, Baylot V, So A and Rocchi P: Hsp27 as a therapeutic target in cancers. Curr Drug Targets 15: 423-431, 2014.

23. Arts HJ, Hollema H, Lemstra W, Willemse PH, De Vries EG, Kampinga $\mathrm{HH}$ and Van der Zee AG: Heat-shock-protein-27 (hsp27) expression in ovarian carcinoma: Relation in response to chemotherapy and prognosis. Int $\mathrm{J}$ Cancer 84: 234-238, 1999.

24. Hadchity E, Aloy MT, Paulin C, Armandy E, Watkin E, Rousson R, Gleave M, Chapet $\mathrm{O}$ and Rodriguez-Lafrasse C: Heat shock protein 27 as a new therapeutic target for radiation sensitization of head and neck squamous cell carcinoma. Mol Ther 17: 1387-1394, 2009.

25. Guo K, Kang NX, Li Y, Sun L, Gan L, Cui FJ, Gao MD and Liu KY: Regulation of HSP27 on NF-kappaB pathway activation may be involved in metastatic hepatocellular carcinoma cells apoptosis. BMC Cancer 9: 100, 2009.

26. Guo K, Liu Y, Zhou H, Dai Z, Zhang J, Sun R, Chen J, Sun Q, $\mathrm{Lu}$ W, Kang $\mathrm{X}$ and Chen P: Involvement of protein kinase $\mathrm{C}$ beta-extracellular signal-regulating kinase 1/2/p38 mitogen-activated protein kinase-heat shock protein 27 activation in hepatocellular carcinoma cell motility and invasion. Cancer Sci 99: 486-496, 2008.

27. Xue L, Yang L, Jin ZA, Gao F, Kang JQ, Xu GH, Liu B, Li H, Wang XJ, Liu LJ, et al: Increased expression of HSP27 inhibits invasion and metastasis in human esophageal squamous cell carcinoma. Tumour Biol 35: 6999-7007, 2014.

28. Pavan S, Musiani D, Torchiaro E, Migliardi G, Gai M, Di Cunto F, Erriquez J, Olivero M and Di Renzo MF: HSP27 is required for invasion and metastasis triggered by hepatocyte growth factor. Int J Cancer 134: 1289-1299, 2014 